\title{
Hod Lipson and Melba Kurman: Driverless: intelligent cars and the road ahead
}

\author{
The MIT Press, 2016, pp 312, ISBN: 9780262035224
}

\author{
Christine Zarges ${ }^{1}$
}

Autonomous driving is quickly becoming the hot topic in research and technology and has the potential to be truly disruptive. With global companies from different sectors such as Google, Uber and Tesla racing ahead, a futuristic world without human drivers suddenly seems to be in arm's reach. However, many technological and non-technological challenges still need to be solved before driverless cars will be commercially available and dominate our streets.

Driverless-Intelligent Cars and the Road Ahead by Hod Lipson and Melba Kurman is a thought-provoking book that takes a non-expert reader with an interest in technology on a fascinating journey through nearly 80 years of car manufacturing, robotics and machine learning research and provides a colourful vision for a potential future. In 312 pages (including 8 pages of references and a useful index) it covers major technological achievements and potential benefits but also risks and necessary technological and non-technological requirements. Many of the chapters are given a personal touch by including anecdotes and detailed accounts of visits to research labs and conferences. The authors also discuss differences between research in an academic context and within big global companies-and the problem of political rivalry.

Overall, the book provides an excellent and well-balanced overview of historical developments, the current state-of-the-art and a colourful, sometimes scary, sometimes amusing and sometimes utopian vision for a driverless future. Have you ever wondered if your pizza could be baked to perfection while being delivered? Do you want to design your own car on your computer and have it present itself autonomously at your door step a week later? Would you mind if taxis examine your entire online and shopping history? Serious open questions such as liability in the case of an accident, the problem of massive job losses in the logistic

Christine Zarges

c.zarges@aber.ac.uk

1 Department of Computer Science, Aberystwyth University, Aberystwyth SY23 3DB, UK 
and transport sector, privacy issues and the necessity to adapt current regulations and laws are discussed in several places throughout the book. The discussion is not always straight to the point, taking several detours into related topics-but this is what makes the book captivating, particularly for a non-expert audience.

The authors start with a general introduction in Chapter 1, discussing how cars have changed our way of living and how driverless cars could help overcome problems caused by an ever increasing traffic density. To date, robots have only really taken over in highly specific industrial jobs and closed world scenarios such as factories and warehouses. Overcoming this limitation by learning how to react to a previously unknown situation is the main challenge for autonomous driving. With new technological advances (and decreasing prices) the vision of a driverless world suddenly seems realistic. The chapter concludes by listing seven delaying myths, often used to argue against driverless cars, which will guide the discussion in the remainder of the book.

Chapter 2 paints a colourful and emerging vision of a driverless world including its potential conveniences and risks. Will there be more or fewer cars in the future? Will driverless cars be more fuel-efficient, reduce traffic congestion and air pollution? Will we commute differently? What will the effects on our social interactions, local businesses and the shape of our cities be? How can synergies with electric cars be used effectively? The authors do not provide any definite answers to those questions (how could they!) but provide lots of thoughts that can lend themselves to lively subsequent discussions.

Chapter 3 focusses on different approaches for transition from human to autonomous driving and how car companies may need to rethink their businesses in the light of recent developments. The authors take the clear position that a 'direct dive' into full autonomy (Google's perspective) should be the way forward and argue that a gradual transition (as usually preferred by the automotive industry) would be unsafe as it inevitably creates the problem of split responsibility. They present a large number of examples and studies to support their argument, but also provide reasons why one may be tempted to think otherwise.

Chapter 4 and 5 deal with technical requirements for driverless cars. While Chapter 4 focusses on the car's operating system including low-level (accelerating, braking and turning) and high level controls (route planning and navigation), Chapter 5 discusses the car's perception system including object recognition and artificial vision, the importance of transparent and reliable standards for driverless cars and the necessity of such standards being controlled by governments.

Chapters 6-8 provide an incredibly detailed account of the history of autonomous driving, its different approaches and how some of these technologies are still used today. Starting in the late 1930s (Chapter 6), the authors tell the story of very early visions of an automated highway and achievements by US car manufactures and researchers, which all required some form of specialised highway infrastructure. Socalled V2X technologies (vehicle-to-vehicle and vehicle-to-infrastructure communication), the focus of Chapter 7, are still very much advocated by the US Department of Transportation today, however, the authors strongly believe that the future lies in smart cars, not smart highways. They argue that smart highways can only really develop their benefits when cars are truly autonomous and thus, they 
believe that current policies are not only a waste of money but in fact hinder future developments. In Chapter 8, they therefore turn their attention to modern driverless cars which have their roots in intelligent robotics and do not rely on any specific infrastructures. Along the way, they shed light on different branches of artificial intelligence and how the use of machine learning contributed to the first breakthroughs.

Chapter 9 focusses on enabling technologies from a hardware perspective while Chapter 10 is dedicated to deep-learning technology, "the crown jewel of the control software that guides a robot's artificial perception and response" (p. 196). Both Chapters 9 and 10 stand out due to their easy-to-follow explanation of the different technologies and how they work and an incredible attention to detail in covering strengths, limits, challenges and major developments (including all the ups and downs of neural network research).

In Chapter 11 the authors turn their attention to challenges of driverless cars, particularly in the context of privacy and ethical questions such as liability. Some of these aspects are very well suited for classroom discussions-the question of how an optimal response in an accident should look like has led to one of the most lively debates I have ever had in a lecture theatre. The authors argue that cars will be able to improve and solve many of the current problems around route planning and traffic prediction autonomously after reaching a critical tipping point.

Chapter 12 concludes by continuing the authors' vision of a driverless world and discussing requirements for turning it into reality.

The authors' view on the future of driverless cars is a very positive, optimistic and enthusiastic one. In several chapters, they present very precise directions for future developments and standards, particularly with respect to policy making. Many suggestions are inspired by related concepts or past developments. Others are supported by results of surveys or research studies. A reader might of course not agree with all of the suggestions and views presented. She might think that being only twice as safe as a human driver is not quite the standard one should be aiming for. She might be more sceptical towards privacy issues and other potential implications on our everyday life. But this is not important-on the contrary. As the authors point out, there is need for a moral code that should be agreed upon by the general public. This book provides an excellent starting point for such a discussion as it is written with a general rather than a specialist audience in mind. By providing an overview of past, present and future developments it provides insights into enabling technologies, potential benefits and risks. Together with a colourful and imaginative picture of a possible future of our world (that admittedly often resembles images from a science fiction movie), it lends itself to detailed further discussions.

Is all of this realistic? Personally, I am not so sure, but in any case, I have very much enjoyed this journey! 